\title{
Phylogenetic and evolutionary analyses of the VP4 gene of $P[9]$ rotaviruses
}

\section{Sharnali Ahmed}

Universiti Malaysia Sabah

\section{Takaaki Yahiro}

Universiti Malaysia Sabah

Nor Amalina Emran

Universiti Malaysia Sabah

Lia Natasha Amit

Universiti Malaysia Sabah

Hidekatsu Iha

Oita Daigaku

Kamruddin Ahmed ( $\square$ ahmed@ums.edu.my )

Universiti Malaysia Sabah https://orcid.org/0000-0002-1869-3701

\section{Research note}

Keywords: Rotavirus, genotype P[9], phylogenetic analysis, reassortant, lineages

Posted Date: October 21st, 2020

DOl: https://doi.org/10.21203/rs.3.rs-43564/v2

License: (c) (i) This work is licensed under a Creative Commons Attribution 4.0 International License.

Read Full License 


\section{Abstract}

Objectives: Rotavirus is one of the major causes of gastroenteritis in children under 5 years of age and is responsible for over 200,000 deaths annually. Rotavirus can evolve by reassortment, in which gene segments are exchanged between strains of different origins. Rotavirus strains with the $\mathrm{P}[9]$ genotype is an example of reassortment, in which the $\mathrm{P}[9]$ genotype is from feline species. A number of outbreaks associated with $\mathrm{P}[9]$ strains have been documented in several countries. However, details regarding the epidemiological relationships between the strains remains largely unknown. Therefore, in the present study, genetic characterization and evolutionary analyses were performed to gain insight into $P[9]$ strains circulating in different parts of the world.

Results: The VP4 gene of the P[9] strains could be divided into six lineages, and P[9] strains characterized in this study share a common ancestor that circulated in circa 1864. In each lineage, the strains were not only from different countries, but also from different continents. These findings suggest that none of the lineages has a specific region of distribution, and although humans have had interactions with cats for thousands of years, the common ancestor of the VP4 gene of the current P[9] strains is relatively recent.

\section{Introduction}

Rotavirus is a major cause of gastroenteritis and is responsible for over 200,000 deaths annually in children under 5 years of age [1]. Rotavirus has a double-stranded RNA genome divided into 11 segments, encoding six structural (VP1-VP4, VP6, and VP7) and six nonstructural proteins (NSP 1-6) [2]. A binary classification system developed on the basis of two outer capsid proteins, VP7 and VP4, using G and P genotypes is used to identify different strains of group A rotavirus. To date $36 \mathrm{G}$ genotypes and $51 \mathrm{P}$ genotypes have been found (https://rega.kuleuven.be/cev/viralmetagenomics/virus-classification/rcwg). Among the numerous G/P-genotype combinations, only a limited number are commonly found in human infections; these are G1P[8], G2P[4], G3P[8], G4P[8], G9P[8], and G12P[8][3].

One of the mechanisms by which rotavirus evolves is reassortment, where segments of genes are exchanged between strains of different origin. Among the known reassortment strains,G3P[9] is the least studied, but has become prominent, where the VP7 G3 gene often detected in associated with P[9] is feline-like as well in many reports[4]. The common $G$ genotypes in combination with $P[9]$ are G3, G6, G1, and $\mathrm{G} 12$ [5-11]. The incidence of P[9] strains causing infection in humans is relatively low, at about $2.5 \%$ worldwide [12]. Despite this low incidence, the prevalence of cases with P[9] strains is higher in several countries [13-23]. In Brazil and Ireland, around $10 \%$ and $18 \%$ of the patients infected with rotavirus had $P[9]$ strains $[21,24]$. The other point of concern is that $P[9]$ strains might become more virulent over time with multiple reassortments have the potential to cause outbreaks in other areas.

Based on limited studies, the evolutionary patterns of the human P[9] rotaviruses appear to be complex [23]. Therefore, the present study was performed to determine the genetic relationships among the VP4 gene of $P[9]$ strains circulating in different countries, and their evolutionary timelines. 


\section{Materials And Methods}

\section{Phylogenetic analyses}

A total of 94 full- and partial-length VP4 gene nucleotide sequences of P[9] strains were extracted from GenBank (Additional file 1). Phylogenetic analyses were conducted with the maximum likelihood method using MEGA X [25] after aligning the nucleotide sequences using CLUSTAL W [26]. The branching patterns were evaluated based on a bootstrap analysis of 1,000 replicates. In all of the phylogenetic trees, lineages were designated based on significant bootstrap values of $>70 \%$. Several phylogenetic trees were constructed using full-length gene and partial-length genes of different lengths (Additional file 2).

\section{Nucleotide identity}

The nucleotide identities of the full- and partial-length P[9] genes of different lineages were calculated by online software (www.bioinformatics.org).

\section{Timeline and evolution}

Evolutionary analysis was performed using the full-length nucleotide sequences of VP4 genes. We inferred a maximum clade credibility phylogenetic tree using the Bayesian Markov Chain Monte Carlo method available in BEAST version 1.6.1 [27]. The nucleotide sequences were analyzed by using a relaxed molecular clock (uncorrelated lognormal) and general time-reverse model (GTR $+1+\Gamma$ model). The sequences were run for 60 million generations and sampled at every 3,000 steps. The end result was a sample size of 2,000 Bayesian trees, which was then verified for convergence by Tracer version 1.5.

\section{Results}

\section{Phylogenetic analyses}

In the phylogenetic tree constructed using 43 full-length gene sequences of the VP4 gene of the P[9] strains, six lineages could be identified (Fig. 1), lineage la, Ib, Ic, Id, le, and II. Lineage la consisted of G3P[9], G1P[9], G3G4P[9], and G12P[9] strain from Paraguay, Japan, China, Thailand and Korea. Paraguayan strains were of genotypes G1P[9], G3P[9], G3G4P[9], and G12P[9]. Strains from other countries were of genotype G3P[9] (Additional file 3). Lineage lb consisted of strains from Italy, Australia, and Japan. All these strains were of genotype G3P[9]. Lineage Ic consisted of G3P[9] and G6P[9] strains from Russia, Japan, Italy, Korea, Hungary, and Tunisia. Among these, strains from Russia, Korea, Hungary, and Italy were G3P[9], while strains from Tunisia and Japan were G6P[9]. Lineage Id consisted of strains from the USA, and Italy; all were G3P[9]. Lineage le consisted of two strains from Lebanon of G3P[9]. Lineage II consisted of strains from Paraguay, Brazil, Italy and Thailand. All of the strains in lineage II were of the G12P[9] combination.

A total of 11 phylogenetic trees were constructed using different partial-length nucleotide sequences (Additional file 2) of P[9] strains. From partial-length nucleotide sequence of 837 and above consistently 
generated trees which could be classified into six lineages. The distribution of strains in each lineage was consistent with the phylogenetic tree constructed using full- and partial-length gene sequences.

Overall, six lineages of $\mathrm{P}[9]$ strains could be identified in the phylogenetic tree constructed using 57 partial gene sequences (43-879 nt) of P[9] strains (Fig. 2). Lineage la consisted of G3P[9], G1P[9], G9P[9], G12P[9], and G3G4P[9] strain from Brazil, Japan, Paraguay, Thailand, China, , and Korea. The strains from Brazil, were of the G3P[9], G1P[9], and G9P[9] combination (Additional file 3). G3P[9] combination was from Japan. Strains from Paraguay were G3P[9], G1P[9], G12P[9], and G3G4P[9]. All strains from Thailand, China, and Korea were G3P[9]. Lineage lb consisted of G3P[9] strains from Italy, Australia and Japan. Lineage Ic consisted of genotype G3P[9], and G6P[9] from Russia, Hungary, Italy, Tunisia, Japan, and Korea. Russian strains were of the G3P[9], and G6P[9] combination. The strains from Hungary was of genotype G3P[9] . Italian strains were of genotype G3P[9] and G6P[9]. The strains from Tunisia and Japan were G6P[9], and from Korea were G3P[9]. Lineage Id consisted of genotype G3P[9] from the USA, and Italy,. Lineage le consisted of strains from Lebanon of genotype G3P[9]. Lineage II consisted of strains from Paraguay, Brazil, Thailand and Italy. All strains were of the G12P[9] combination.

\section{Nucleotide identity}

Comparison of nucleotide identities of 43 full-length and 57 partial-length nucleotide sequences of the outer capsid protein VP4 gene among the six lineages of rotavirus P[9] are shown in additional file 4 and 5 , respectively.

Full-length nucleotide sequences of P[9] strains of the lineage la, lb, Ic, Id, le, and II shared nucleotide identity of $96.2 \%-100 \%, 97.6 \%-99.5 \%, 96.2 \%-99.7 \%, 97.8 \%, 96.6 \%$, and $97.2 \%-99.9 \%$ among themselves, respectively. The strains of different lineages showed a decrease in nucleotide identity $(91.9 \%-99.7 \%)$, except for lineage II, which shared relatively low nucleotide identity $(88.5 \%-90.5 \%)$ with strains of other lineages.

Partial-length nucleotide sequences of P[9] strains of the lineage la, lb, Ic, Id, le, and II shared nucleotide identity of $95.5 \%-100 \%, 98.2 \%-99.2 \%, 96.2 \%-100 \%, 97.9 \%, 97.5 \%$, and $97.0 \%-100 \%$ among themselves, respectively. The strains of different lineages showed a decrease in nucleotide identity $(92.5 \%-96.3 \%)$, except for lineage II, which shared relatively low nucleotide identity $(88.2 \%-90.8 \%)$ with strains of other lineages.

\section{Timeline of evolution}

The phylogenetic tree constructed using the Bayesian method also showed six lineages of P[9] strains (Fig. 3), which corresponded with the lineages as determined using the maximum likelihood method. All $\mathrm{P}[9]$ included in this study shared a common ancestor in circa 1864 (95\% highest posterior density [HPD] circa 1755-1941) when lineage II diverged from lineage la, Ib, Ic, Id, and le. The strains in lineage II started evolving in circa 1983 (95\% HPD 1964-1993). Lineage le diverged from lineages la, Ib, Ic, and Id 
in circa 1932 (95\% HPD 1896-1960). Lineage la diverged from lineages lb, lc, and Id in circa 1945 (95\% HPD 1919-1964) and different strains of lineage la evolved in circa 1969 (95\% HPD 1958-1977). Lineage Id diverged from lineage lb, and Ic in circa 1948 (95\% HPD 1923-1966 years). Lineage lb diverged from lineage Ic in circa 1953 (95\% HPD 1929-1970), and the strains in lineage lc started evolving in circa 1983 (95\% HPD 1969-1994).

\section{Discussion}

All currently circulating P[9] strains were divided into six lineages, except for lineage le, the strains in each lineage were from multiple countries on different continents. This suggests that strains in a lineage do not belong to a specific geographical area. This might exemplify the role of human migration in the spread of strains in different countries. Humans tend to bring their accompanying animals, which might include domestic cats, during migration, and as human migration is a continuous process, this trend is expected to continue in future.

The results of this study confirmed sequences of the VP4 gene as short as $837 \mathrm{nt}$ were adequate for lineage designation. For new strains, it is still recommended where possible to use full-length nucleotide sequences for phylogenetic analysis to provide a more robust and comprehensive for analysis of the evolution, spread, and genome-wide heterogeneity of a given virus. In the present study, full-length nucleotide sequences were used during timeline evolutionary analysis because different portions of the gene have different rates of evolution, which might affect the determination of lineage age.

Lineage la contained the AU-1 strain (G3P[9]), which was the first detected P[9] strain in humans [9]. The Brazilian strains were from several outbreaks [21]; these strains were also clustered in this lineage and shared high nucleotide identity with AU-1 [28]. All Paraguayan P[9] strains of lineage la were detected in the same year; however, the $\mathrm{P}[9]$ genotype was in combination with different $\mathrm{G}$ genotypes. By contrast, the Paraguayan strains of lineage II were all G12P[9]. These were considered emerging strains [19], suggesting the possibility of outbreak. The results of the present study support the finding that Paraguayan strains share $99 \%$ nucleotide identity with $\mathrm{T} 152$, another $\mathrm{G} 12 \mathrm{P}[9]$ rotavirus strain discovered in Thailand [10]. However, the underlying mechanism for the specific combination of the P[9] from lineage II with $\mathrm{G} 12$ rather than $\mathrm{G} 3$ requires further study.

When full nucleotide sequences were compared, the $P[9]$ strains of the same lineage shared close nucleotide identity among themselves. When strains of different lineages were compared, a decrease in nucleotide identity was observed, except for lineage II, which shared relatively low nucleotide identity with strains of other lineages. All of the strains in lineage II were G12P[9]; the significance of this genotype combination on nucleotide identity requires further study. Also, five of eight strains in lineage II were from South America and formed a cluster, which suggests the possibility of strains descended from a single ancestor are spreading across the continent. Few differences were seen when the identities of partiallength sequences were compared with those of full-length sequences. Such differences in nucleotide 
identity using partial-length nucleotide sequences might be acceptable when no full-length sequences are available.

The information obtained in this study indicates that the origin of the common ancestor of currently circulating P[9] rotavirus strains might be too recent. We postulate that there could have been several rotavirus transmission events from cats to humans; however, older strains might have been wiped out by evolutionary constraints, and the currently circulating strains that evolved from a common ancestor in circa 1864 which could have survived and dispersed in different places with further local evolution. Because of increased human-animal interaction in recent decades it is possible that the current strains might evolve further and give rise to virulent strains of rotavirus.

Human-animal interaction has increased in recent years for several reasons, such as more humans having pets, a loss of animal habitats because of deforestation, and increase in large-scale farming and ecotourism. As a result, the potential for zoonotic transmission of viruses has increased, which could lay the foundation for the emergence of reassorted strains. The development of a common P[9] vaccine for humans and cats might help control rotavirus infection by this reassorted strain.

We conclude that the VP4 gene of the available P[9] strains could be divided into six lineages. VP4 gene as short as $837 \mathrm{nt}$ were adequate for lineage designation. Although humans have had interactions with cats for thousands of years, the common ancestor of the current $P[9]$ strain is relatively recent. We also found that none of the lineages has a specific region of distribution.

\section{Limitations}

- Further study is needed particularly using nucleotide sequences of $\mathrm{P}[9]$ strains from cats to evaluate the time-line of evolution of $\mathrm{P}[9]$ strains.

- Many of the genes in your analysis were sequenced from strains that have undergone passage in cell culture which might had impact on the analyses.

\section{Abbreviations}

RNA: Ribonucleic acid; MEGA: Molecular evolutionary genetics analysis; BEAST: Bayesian evolutionary analysis by sampling trees; GTR: General time reverse; USA: United States of America; HPD: Highest posterior density; Nt: Nucleotide.

\section{Declarations}

\section{Ethics approval and consent to participate}

Not applicable

\section{Consent to publish}


Not applicable

\section{Availability of data and materials}

The datasets used and/or analyzed during the current study are available in the GenBank. These are also available from the corresponding author on reasonable request.

\section{Competing interests}

The authors declare that they have no competing interests.

\section{Funding}

This study was supported in part by a grant, FRGS-0457-2017, from the Ministry of Higher Education, Malaysia.

\section{Authors' contributions}

KA conceived and designed the experiments. NAE and KA performed general supervision and guidance in the research process. SA TY LNA KA performed the experiments. SA TY NAI LNA HI analyzed and interpreted the data. SA TY NAI LNA HI drafted the manuscript. All authors read and approved the final manuscript.

\section{Acknowledgments}

Not applicable

\section{References}

1. Tate JE, Burton AH, Boschi-Pinto C, Parashar UD: Global, Regional, and National Estimates of Rotavirus Mortality in Children <5 Years of Age, 2000-2013. Clin Infect Dis. 2016, 62 Suppl 2:S96S105.

2. Matthijnssens J, Ciarlet M, Rahman M, Attoui H, Banyai K, Estes MK, Gentsch JR, Iturriza-Gomara M, Kirkwood CD, Martella $V$ et al: Recommendations for the classification of group A rotaviruses using all 11 genomic RNA segments. Arch Virol. 2008, 153:1621-1629.

3. Bibera GL, Chen J, Pereira P, Benninghoff B: Dynamics of G2P[4] strain evolution and rotavirus vaccination: A review of evidence for Rotarix. Vaccine 2020, 38:5591-5600.

4. Nakagomi T, Nakagomi O: RNA-RNA hybridization identifies a human rotavirus that is genetically related to feline rotavirus. J Virol. 1989, 63:1431-1434.

5. De Grazia S, Giammanco GM, Doro R, Bonura F, Marton S, Cascio A, Martella V, Banyai K: Identification of a multi-reassortant G12P[9] rotavirus with novel VP1, VP2, VP3 and NSP2 genotypes in a child with acute gastroenteritis. Infect Genet Evol. 2015, 35:34-37. 
6. Isegawa Y, Nakagomi O, Nakagomi T, Ueda S: A VP4 sequence highly conserved in human rotavirus strain AU-1 and feline rotavirus strain FRV-1. J Gen Virol. 1992, 73:1939-1946.

7. Mladenova Z, Nawaz S, Ganesh B, Iturriza-Gomara M: Increased detection of G3P[9] and G6P[9] rotavirus strains in hospitalized children with acute diarrhea in Bulgaria. Infect. Genet Evol. 2015, 29:118-126.

8. Nakagomi O, Nakagomi T: Interspecies transmission of rotaviruses studied from the perspective of genogroup. Microbiol Immunol. 1993, 37:337-348.

9. Nakagomi O, Ohshima A, Aboudy Y, Shif I, Mochizuki M, Nakagomi T, Gotlieb-Stematsky T: Molecular identification by RNA-RNA hybridization of a human rotavirus that is closely related to rotaviruses of feline and canine origin. J Clin Microbiol. 1990, 28:1198-1203.

10. Pongsuwanna Y, Guntapong R, Chiwakul M, Tacharoenmuang R, Onvimala N, Wakuda M, Kobayashi $\mathrm{N}$, Taniguchi K: Detection of a human rotavirus with G12 and P[9] specificity in Thailand. J Clin Microbiol. 2002, 40:1390-1394.

11. Yamamoto D, Kawaguchiya M, Ghosh S, Ichikawa M, Numazaki K, Kobayashi N: Detection and full genomic analysis of G6P[9] human rotavirus in Japan. Virus Genes. 2011, 43:215-223.

12. Khamrin P, Maneekarn N, Peerakome S, Tonusin S, Phan TG, Okitsu S, Ushijima H: Molecular characterization of rare G3P[9] rotavirus strains isolated from children hospitalized with acute gastroenteritis. J Med Virol. 2007, 79:843-851.

13. Ben Hadj Fredj M, Heylen E, Zeller M, Fodha I, Benhamida-Rebai M, Van Ranst M, Matthijnssens J, Trabelsi A: Feline origin of rotavirus strain, Tunisia, 2008. Emerg Infect Dis. 2013, 19:630-634.

14. De Grazia S, Giammanco GM, Martella V, Ramirez S, Colomba C, Cascio A, Arista S: Rare AU-1-like G3P[9] human rotaviruses with a Kun-like NSP4 gene detected in children with diarrhea in Italy. J Clin Microbiol 2008, 46:357-360.

15. De Grazia S, Giammanco GM, Potgieter CA, Matthijnssens J, Banyai K, Platia MA, Colomba C, Martella V: Unusual assortment of segments in 2 rare human rotavirus genomes. Emerg Infect Dis 2010, 16:859-862.

16. Gomez MM, Resque HR, Volotao Ede M, Rose TL, da Silva MF, Heylen E, Zeller M, Matthijnssens J, Leite JP: Distinct evolutionary origins of G12P[8] and G12P[9] group A rotavirus strains circulating in Brazil. Infect Genet Evol. 2014, 28:385-388.

17. Grant L, Esona M, Gentsch J, Watt J, Reid R, Weatherholtz R, Santosham M, Parashar U, O'Brien K: Detection of G3P[3] and G3P[9] rotavirus strains in American Indian children with evidence of gene reassortment between human and animal rotaviruses. J Med Virol. 2011, 83:1288-1299.

18. Jeong S, Than VT, Lim I, Kim W: Whole-genome analysis of a rare human Korean G3P rotavirus strain suggests a complex evolutionary origin potentially involving reassortment events between feline and bovine rotaviruses. PLoS One 2014, 9:e97127.

19. Martinez M, Amarilla AA, Galeano ME, Aquino VH, Farina N, Russomando G, Parra GI: Predominance of rotavirus G2P[4] and emergence of G12P[9] strains in Asuncion, Paraguay, 2006-2007. Arch Virol. 2010, 155:525-533. 
20. Nguyen TH, Than VT, Thanh HD, Kim W: Evidence of multiple reassortment events of feline-to-human rotaviruses based on a rare human G3P[9] rotavirus isolated from a patient with acute gastroenteritis. Compar Immunol Microbiol Infect Dis. 2016, 46:53-59.

21. Santos N, Volotao EM, Soares CC, Albuquerque MC, da Silva FM, de Carvalho TR, Pereira CF, Chizhikov V, Hoshino Y: Rotavirus strains bearing genotype G9 or P[9] recovered from Brazilian children with diarrhea from 1997 to 1999. J Clin Microbiol 2001, 39:1157-1160.

22. Theamboonlers $A$, Maiklang $O$, Thongmee $T$, Chieochansin $T$, Vuthitanachot $\mathrm{V}$, Poovorawan $\mathrm{Y}$ : Complete genome analysis of a rare human G3P[9] rotavirus posing as an AU-1 like strain. Springer Plus 2013, 2:569.

23. Wang YH, Pang BB, Zhou X, Ghosh S, Tang WF, Peng JS, Hu Q, Zhou DJ, Kobayashi N: Complex evolutionary patterns of two rare human G3P[9] rotavirus strains possessing a feline/canine-like H6 genotype on an AU-1-like genotype constellation. Infect Genet Evol. 2013, 16:103-112.

24. Lennon G, Reidy N, Cryan B, Fanning S, O'Shea H: Changing profile of rotavirus in Ireland: predominance of $P[8]$ and emergence of $P[6]$ and $P[9]$ in mixed infections. J Med Virol. 2008, 80:524530 .

25. Kumar S, Stecher G, Li M, Knyaz C, Tamura K: MEGA X: Molecular Evolutionary Genetics Analysis across Computing Platforms. Mol Biol Evol. 2018, 35:1547-1549.

26. Tamura K, Peterson D, Peterson N, Stecher G, Nei M, Kumar S: MEGA5: molecular evolutionary genetics analysis using maximum likelihood, evolutionary distance, and maximum parsimony methods. Mol Biol Evol. 2011, 28:2731-2739.

27. Drummond AJ, Rambaut A: BEAST: Bayesian evolutionary analysis by sampling trees. BMC Evol Biol 2007, 7:214.

28. Tsugawa T, Rainwater-Lovett K, Tsutsumi H: Human G3P[9] rotavirus strains possessing an identical genotype constellation to AU-1 isolated at high prevalence in Brazil, 1997-1999. J Gen Virol. 2015, 96:590-600.

\section{Figures}




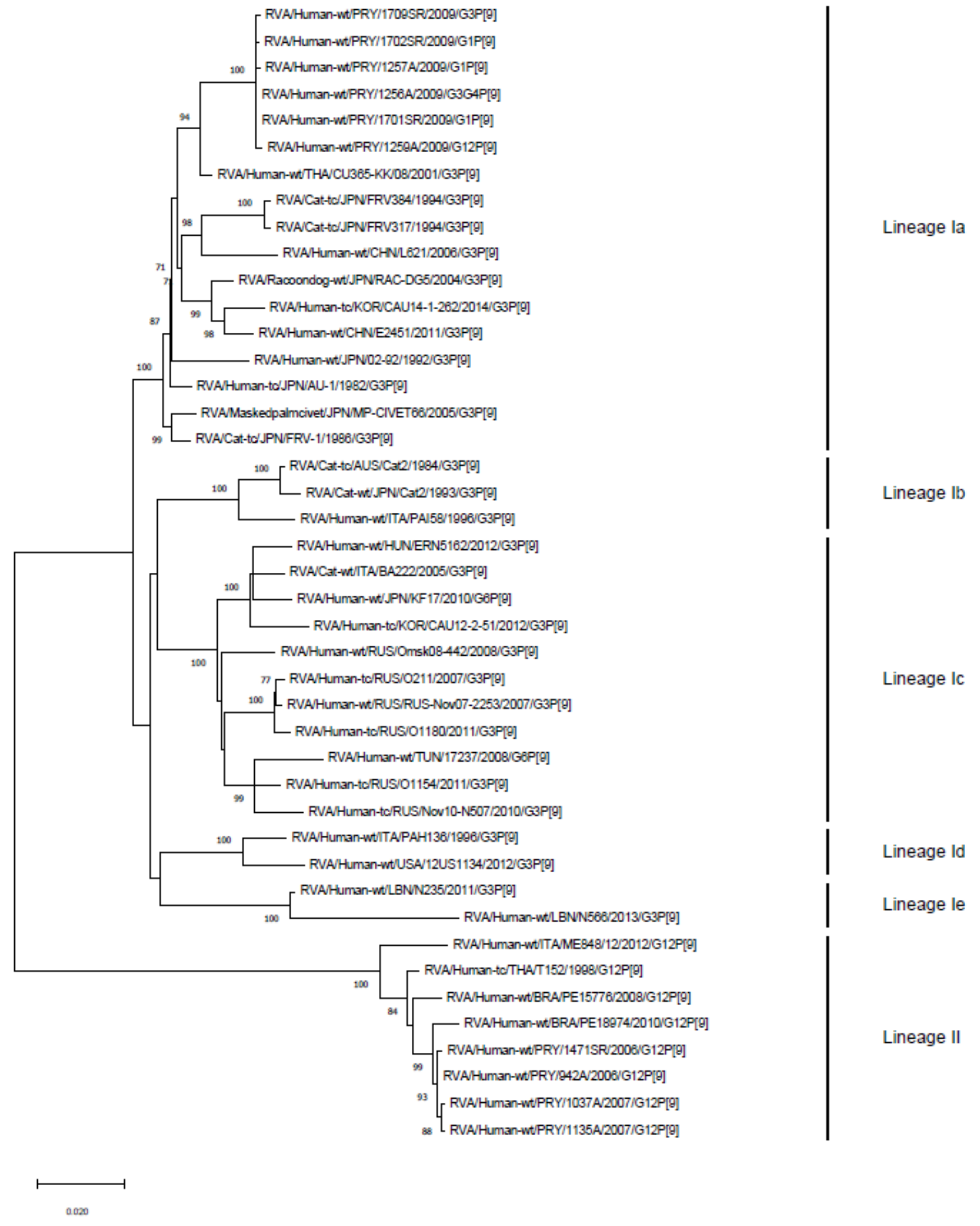

\section{Figure 1}

Phylogenetic tree constructed based on the full nucleotide sequences of the outer capsid protein VP4 genes of P[9] rotavirus strains. The numbers adjacent to the nodes represent the bootstrap values; values $<70 \%$ are not shown. The scale bar shows genetic distance, which is expressed as nucleotide substitution per site. 


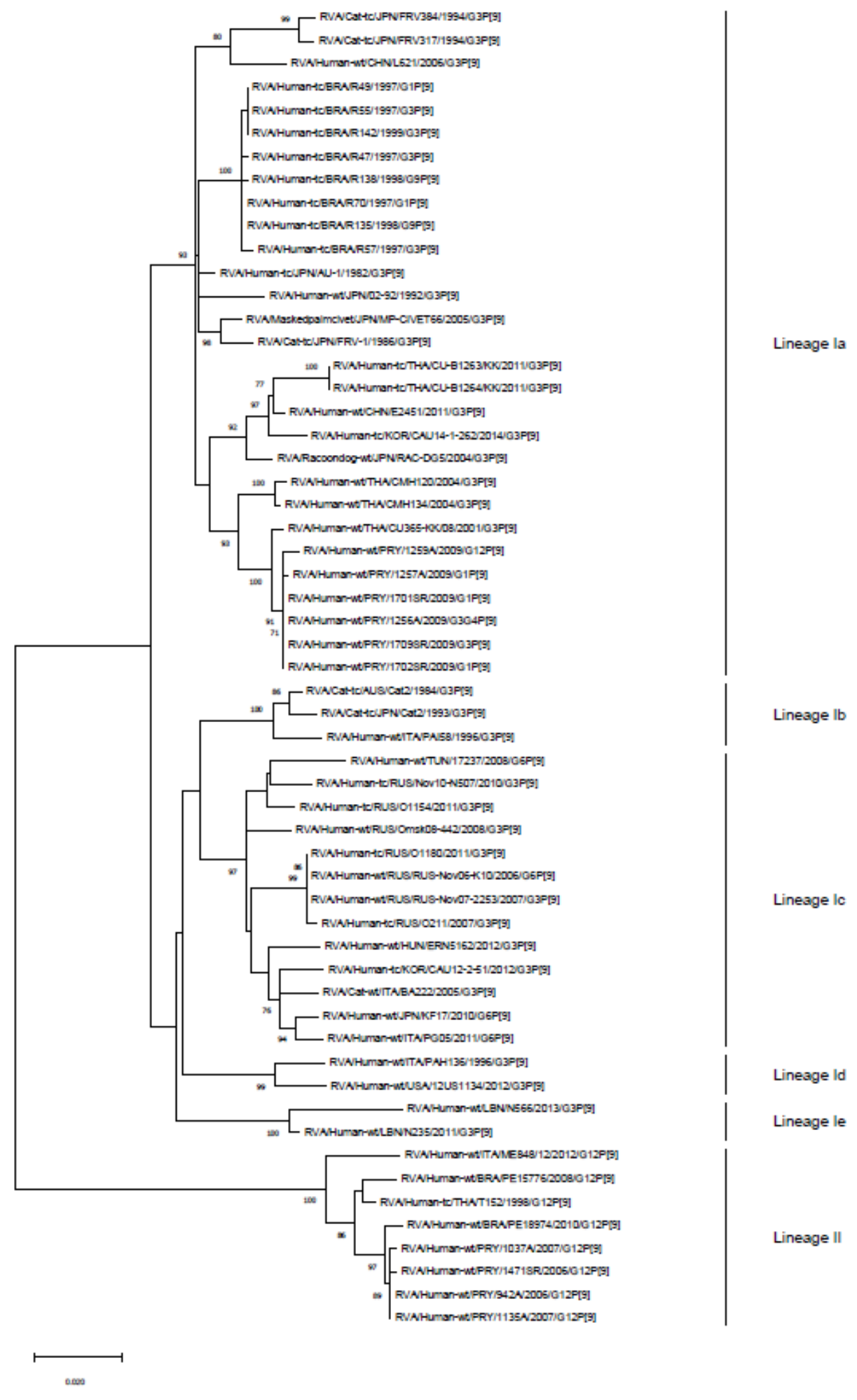

\section{Figure 2}

Phylogenetic tree constructed based on the partial nucleotide sequences of the outer capsid protein VP4 genes of P[9] rotavirus strains, with a length of 837 nucleotides. The numbers adjacent to the nodes represent the bootstrap values; values $<70 \%$ are not shown. The scale bar shows genetic distance, which is expressed as nucleotide substitution per site. 


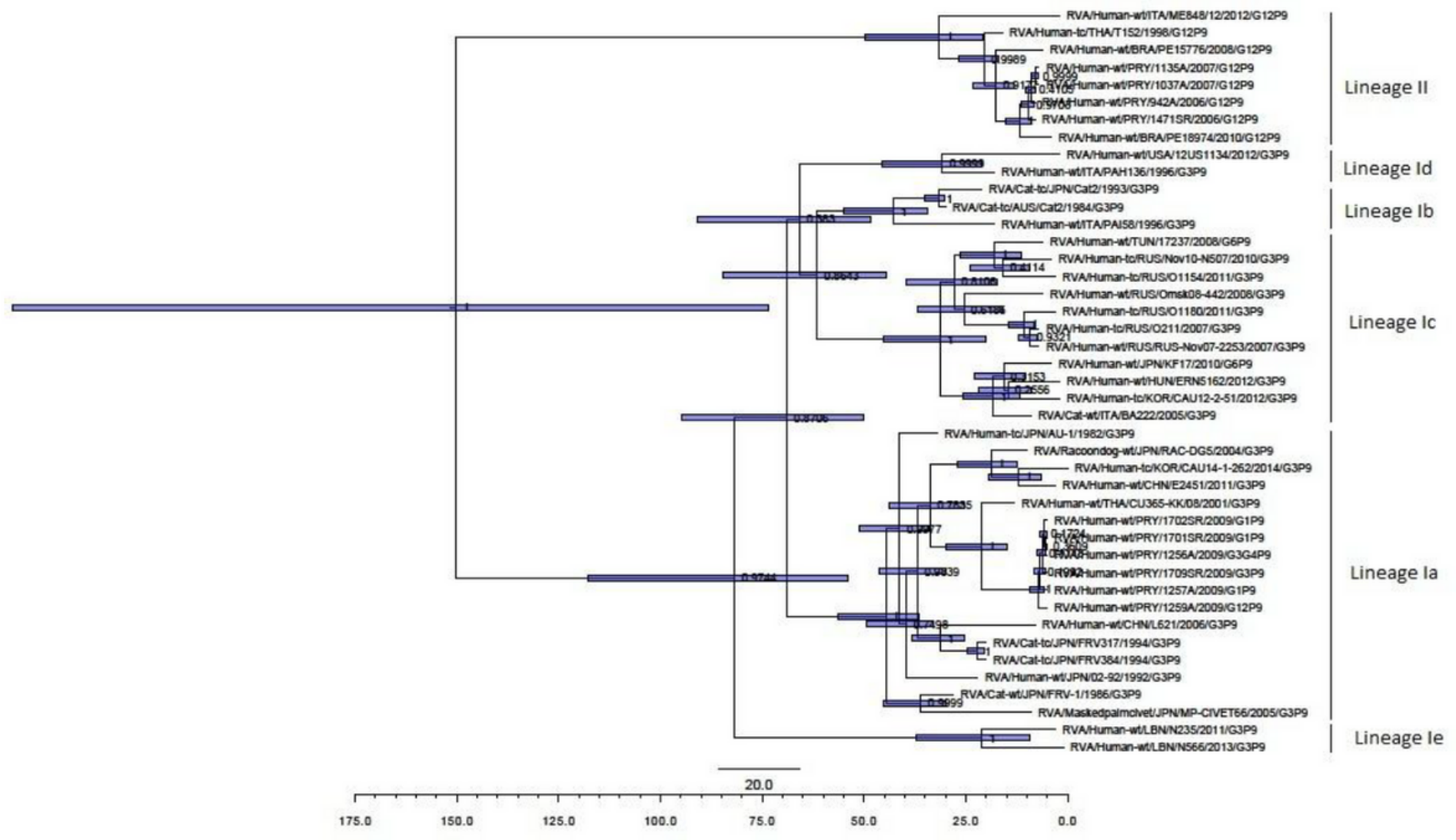

\section{Figure 3}

Bayesian maximum credibility tree showing the ancestry of rotavirus obtained by analyzing full sequences of the outer capsid protein VP4 gene of P[9] rotavirus strains. The nodes correspond to mean age at which the lineages were separated from their most common recent ancestor; blue horizontal bars at the nodes represent the $95 \%$ highest posterior density of the most common recent ancestor. The numbers at the main nodes represent posterior values. The horizontal-axis at the bottom represents the time scale in years.

\section{Supplementary Files}

This is a list of supplementary files associated with this preprint. Click to download.

- Additionalfile120201012.docx

- AdditionalFile220201012.docx

- AdditionalFile320201012.docx

- Additionalfile420201012.docx

- Additionalfile520201012.docx 